K. Sugano (Editor in Chief)

H. Yamamoto, H. Kita (Eds.)

Double-Balloon Endoscopy

Theory and Practice 
K. Sugano (Editor in Chief)

H. Yamamoto, H. Kita (Eds.)

\section{Double-Balloon Endoscopy}

\section{Theory and Practice}


Kentaro Sugano, M.D., Ph.D.

Professor, Department of Internal Medicine

Division of Gastroenterology, Jichi Medical University

3311-1 Yakushiji, Shimotsuke, Tochigi 329-0498, Japan

Hironori Yamamoto, M.D., Ph.D.

Associate Professor, Department of Internal Medicine

Division of Gastroenterology, Jichi Medical University

3311-1 Yakushiji, Shimotsuke, Tochigi 329-0498, Japan

Hiroto Kita, M.D., Ph.D.

Assistant Professor, Department of Internal Medicine

Division of Gastroenterology, Jichi Medical University

3311-1 Yakushiji, Shimotsuke, Tochigi 329-0498, Japan

Library of Congress Control Number: 2006928615

ISBN10 4-431-30204-4 Springer-Verlag Tokyo Berlin Heidelberg New York

ISBN13 978-4-431-30204-5 Springer-Verlag Tokyo Berlin Heidelberg New York

Printed on acid-free paper

This work is subject to copyright. All rights are reserved, whether the whole or part of the material is concerned, specifically the rights of translation, reprinting, reuse of illustrations, recitation, broadcasting, reproduction on microfilms or in other ways, and storage in data banks.

The use of registered names, trademarks, etc. in this publication does not imply, even in the absence of a specific statement, that such names are exempt from the relevant protective laws and regulations and therefore free for general use.

Product liability: The publisher can give no guarantee for information about drug dosage and application thereof contained in this book. In every individual case the respective user must check its accuracy by consulting other pharmaceutical literature.

Springer is a part of Springer Science+Business Media

springer.com

CKentaro Sugano, Hironori Yamamoto, Hiroto Kita 2006

Printed in Japan

This English translation is based on the Japanese original

K. Sugano, H. Yamamoto, H. Kita: Double-Balloon Endoscopy

Published by Nankodo Co., Ltd.

C2005 Kentaro Sugano, Hironori Yamamoto, Hiroto Kita

Typesetting, printing and binding: Kato Bunmeisha, Japan 


\section{Preface}

The double-balloon endoscope came about as the combined result of a brilliant idea and tenacious effort by Hironori Yamamoto, an associate professor in our department. The first clinical application of the double-balloon method was made for diagnosis of hemangioma in the small intestine of a patient with Maffucci's syndrome, as described in this book. Dr. Yamamoto attached a handmade balloon to an upper gastrointestinal endoscope and manually inflated and deflated the balloon, with a sphygmomanometer monitoring the pressure. The maneuverability and the time required for examination appeared to be similar to those in the procedure using a conventional enteroscope, although I was impressed by the fact that double-balloon endoscopy actually revealed hemangioma previously suspected by contrast radiogram, validating the concept of the double-balloon method. However, there were various problems with the handmade device, including balloon damage, dropout, and air leakage, as well as the patience required on the part of the assistant doctors, all of which made its practical application difficult. Despite these circumstances, Dr. Yamamoto clearly identified and solved the problems one by one. As he describes in chapter 1 of this book, the cooperation of manufacturers such as Nisco and Fujinon was a great help, and many medical staff in our department who continuously assisted him in time-consuming examinations also contributed significantly to the practical application of this novel modality of endoscopy. Through the development of this procedure, we directly observed many previously unrecognized lesions and performed endoscopic treatments that had been possible only by surgical operation, and we found that the procedure was tremendously useful for a variety of disorders. Now the double-balloon endoscopy system distributed by Fujinon is in clinical use worldwide and contributes to the diagnosis and treatment of disorders in the small intestine and other regions of the gastrointestinal tract that were not easily accessible by conventional approaches. However, there was no guidebook for systematic training in this procedure, and we believed it was important to provide an opportunity for many clinicians to learn the procedure and perform it properly; thus we decided to publish this book. Consequently, the book provides a range of techniques and tips developed through trial and error by Dr. Yamamoto and our colleagues, who are co-authors of the book. A DVD is included to provide a visual presentation of specific manipulations.

As described in the book, the double-balloon method was initially intended for use in enteroscopy; however, the double-balloon endoscope allows observation of the entire gastrointestinal tract, including the stomach and the large intestine. In addition, the endoscope allows shortening and straightening of the intestinal tract and is suitable for examination of a reconstituted intestine after Roux-en-Y anastomosis and colonoscopy in which insertion is difficult. Thus, we have changed the initially planned title Double-Balloon Enteroscopy to Double-Balloon Endoscopy.

Some people were skeptical of the clinical significance of the procedure, because intestinal diseases were previously considered to be uncommon and the development of the double-balloon endoscope paralleled that of the capsule endoscope. As evidenced by our shortterm experience described in this book, however, we realized that a variety of lesions, hitherto unrecognized because of their limited accessibility, were present in the small intestine as well as in the blind loop. Compared with the capsule endoscope, the double-balloon endoscope produces images with much higher resolution, provides maneuverability that allows retrograde examination, and permits concomitant use of a variety of diagnostic tech- 
niques such as biopsy, dye spraying, and contrast-enhanced examination. It should also be noted that the double-balloon endoscope is compatible with almost all procedures for endoscopic treatment. In combination with those procedures, double-balloon endoscopy now appears to be far superior to capsule endoscopy with respect to diagnostic and therapeutic performance.

This book is intended to be useful as a technical manual and does not include details of individual diseases and the basis for their diagnosis. It may be somewhat difficult for complete novices to understand the initial settings and other technical details that we are accustomed to. In the future, we will make revisions in response to questions and criticisms from readers. We hope, however, that the book will contribute to the elucidation of the pathophysiological mechanisms of intestinal diseases and their treatments, which we believe will evolve dramatically in the twenty-first century with the general availability of doubleballoon endoscopy.

\author{
Kentaro Sugano \\ Department of Internal Medicine \\ Division of Gastroenterology \\ Jichi Medical University, Japan \\ January 2006
}




\section{Contents}

2.1 Configuration 5

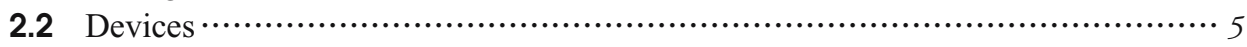

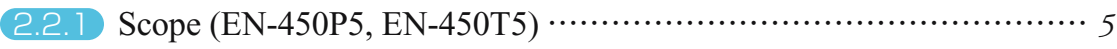

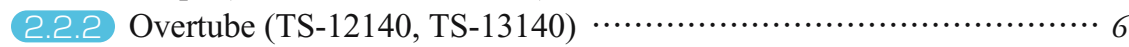

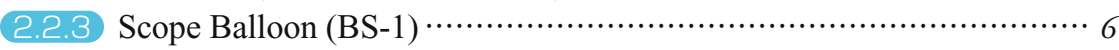

2.2.4 Balloon Pump Controller (PB-20) ……......................... 7

Indications, Contraindications, and Preoperative Examination

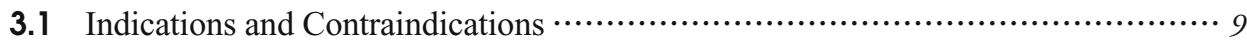

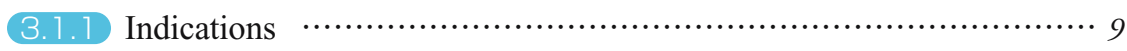

3.1.2 Contraindications ……............................................. 11

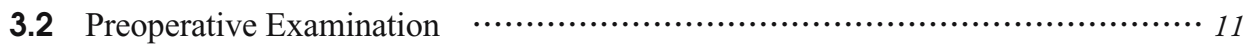

Informed Consent Y. Sekine

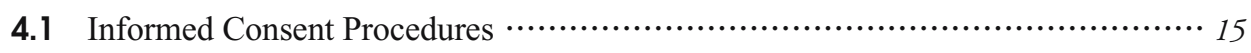

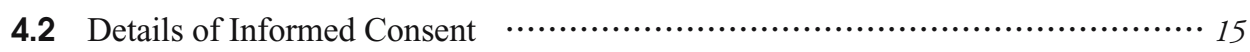

4.2.1) Objective …........................................................ 15

4.2.2 Procedures ……....................................................... 18

4.2.3 Complications ….............................................. 18

4.2.4 Patients' Human Rights ......................................... 18

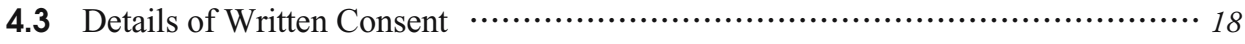

5.1 Principle of Insertion of the Double-Balloon Endoscope …....................... 19

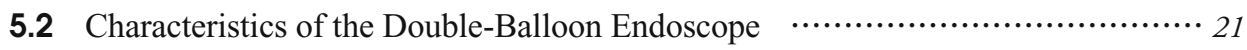




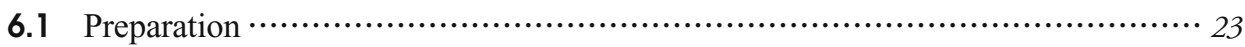

6. 1.1 Double-Balloon Endoscopy Through the Mouth …….................. 23

6. 1.2 Double-Balloon Endoscopy Through the Anus ……...................... 23

6. 1.3 Postoperative Stomach ……........................................... 24

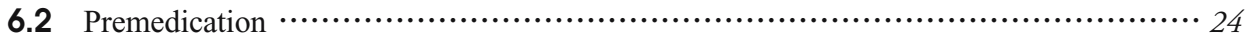

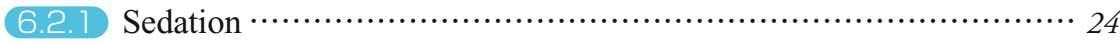

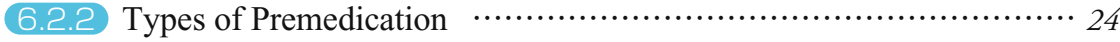

6.2.3 Dosage and Administration ……................................... 25

6.2.4 Monitoring ……...................................................... 25

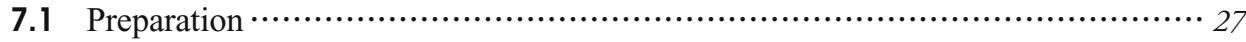

7.2 Points to Consider About Preparation …….................................... 32

8.1 General Considerations for Endoscopy ……........................................... 35

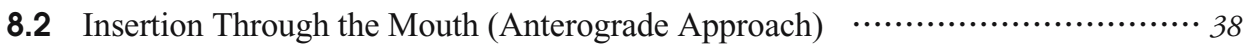

8.3 Insertion Through the Anus (Retrograde Approach) …........................... 40

8.4 Manipulation in the Distal Small Intestine ….................................... 42

\section{Expected Complications and Procedures} to Address the Complications

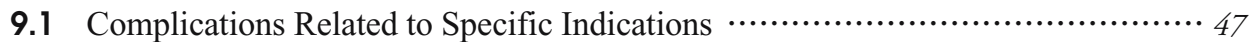

9.2 Complications Related to the Overtube ……................................... 47

9.3 Complications Related to Balloons …….......................................4 48

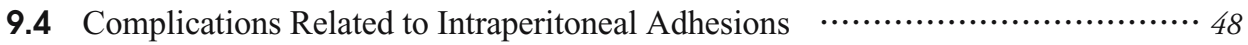

9.5 Complications Associated with Selective Contrast-Enhanced Radiography …..... 48

9.6 Complications Associated with Endoscopic Treatment …......................... 49

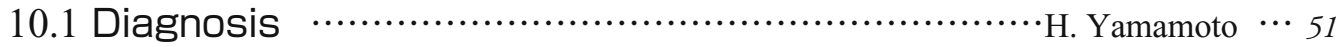

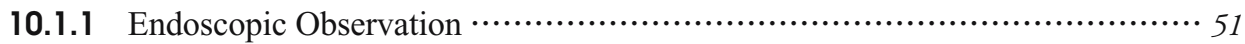

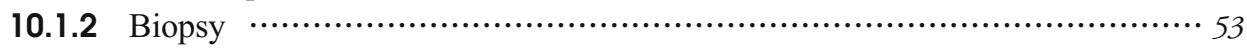

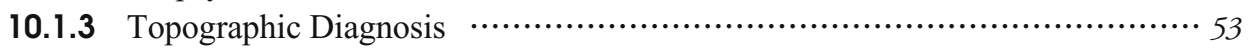

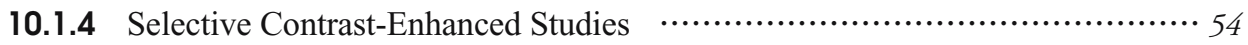


10.1.5 Other Studies

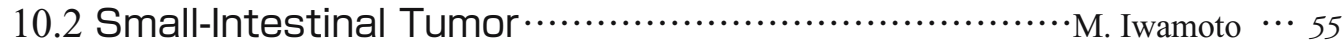

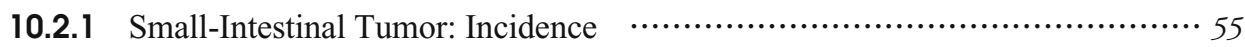

10.2.2 Small-Intestinal Tumors Revealed by Double-Balloon Endoscopy …........... 56

10.2.2. Gastrointestinal Stromal Tumor ……............................ 56

10.2.2.2 Inflammatory Fibroid Polyp ……............................... 57

10.2.2.3 Adenocarcinoma …............................................... 58

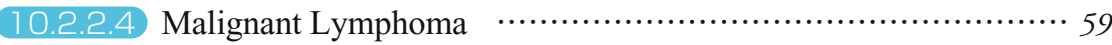

10.2.2.5 Metastatic Large Cell Carcinoma …................................ 60

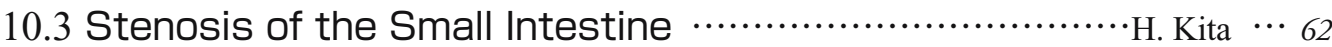

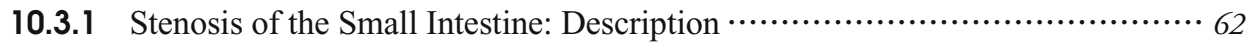

10.3.2 Bowel Obstruction of the Small Intestine ….................................. 62

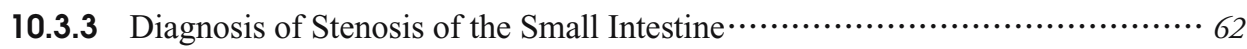

10.3.4 Double-Balloon Endoscopy for Stenosis of the Small Intestine ……............ 63

10.3.5 Endoscopic Treatment of Stenosis of the Small Intestine ….................... 64

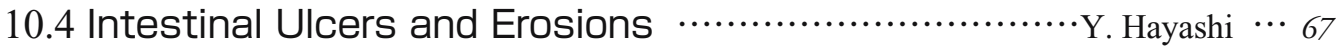

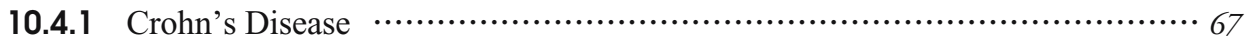

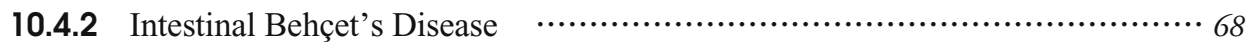

10.4.3 NSAID-Induced, Small-Intestinal Ulcer ….................................... 69

10.4.4 Meckel's Ulcer ……........................................................ 70

10.4.5 Multiple Hemorrhagic Erosions ……...................................... 71

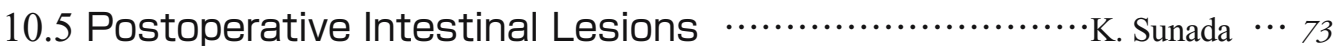

10.5.1 Blind Loop Syndrome ……................................................. 73

10.5.2 Postoperative Adhesive Intestinal Obstructions …............................ 75

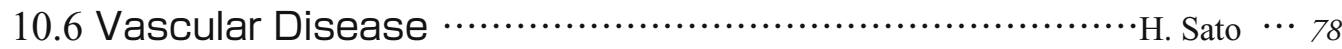

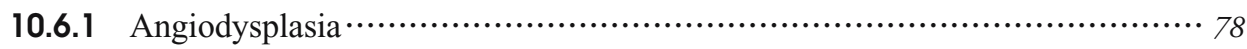

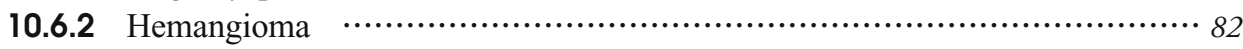

\subsection{Characteristics and Techniques of Endoscopic Treatment}

11.1.1 Characteristics ……....................................................... 83

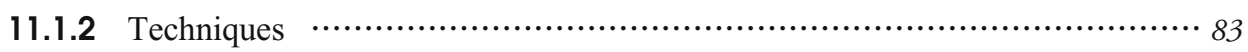

11.1.3 Therapeutic Devices Compatible with the Double-Balloon Endoscope ……. 84 1 1. 1.3.1 Therapeutic Devices for EN-450P5 ……...................... 84

1 1.1.3.2 Representative Useful Therapeutic Devices for Use with EN-450T5 …........................................ 86

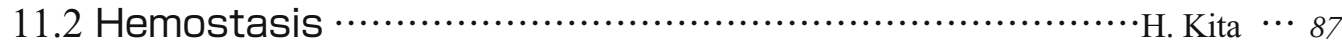

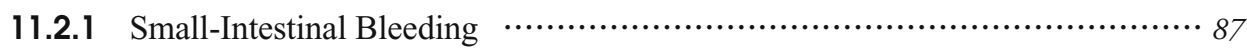

11.2.2 Endoscopic Treatment of Small-Intestinal Bleeding …….................... 87

11.3 Polypectomy and Endoscopic Mucosal Resection $\cdots$ K. Sunada $\cdots 90$

11.3.1 Indications 


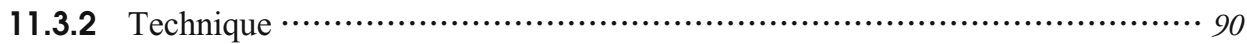

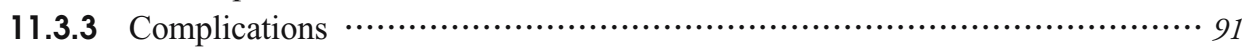

11.3.4 Methods for Collecting Resected Lesions ….................................. 91

1 1 1.3.4.1 Suction …....................................................... 91

1 1.3.4.2 Basket Forceps and Retrieval Net ….............................. 91

11.3.5 Case Presentation …......................................................... 92

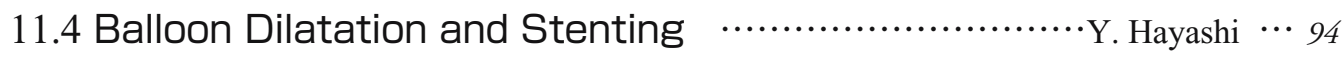

11.4.1 Balloon Dilatation of the Stenotic Site by the Endoscope

Removal Method: Procedure …….......................................... 95

11.4.2 Application to Stenting for Stenosis …...................................... 98

11.4.3 Retrieval of Dropped Hood …............................................ 100

11.4.4 Preparation of the Plastic Tube …......................................... 100

12.1 Double-Balloon Endoscopy as Colonoscopy $\cdots \cdots \cdots \cdots \cdots \cdots \cdots \cdot$ T. Yano $\cdots 101$

12.1.1 Difficult Colonoscopy …...................................................... 101

12.1.2 Applications in the Large Intestine Other than Technically Difficult Cases $\cdots 105$

12.2 Use in Patients with Previous Intestinal Surgery …… T. Yano $\cdots 107$

12.2.1 Specific Tips for Insertion in Patients with a Postoperative Intestine …….... 107

12.2.2 Investigation of the Cause of Bowel Obstruction ….......................... 110

\subsection{Removal of Foreign Bodies from the Small Intestine}

12.3.1 Removal of Parasites from the Small Intestine …............................. 112

12.3.2 Removal of Capsule Endoscope ….................................... 113

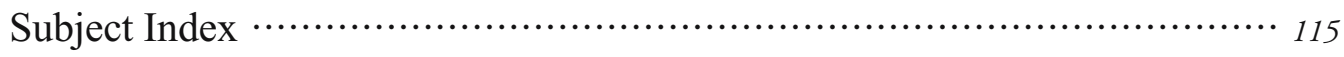




\section{List of Editors and Authors}

Editor in Chief

Kentaro Sugano

\section{Editors}

Hironori Yamamoto

Hiroto Kita

\section{Authors}

Hironori Yamamoto

Masayuki Takano

Syuuichi Yamataka

Tomohiko Miyata

Yutaka Sekine

Tomonori Yano

Keijiro Sunada

Yoshikazu Hayashi

Hiroyuki Sato

Toshihiko Higashizawa

Michiko Iwamoto

Hiroto Kita
Professor

Department of Gastroenterology, Jichi Medical University

Associate Professor

Department of Gastroenterology, Jichi Medical University

Assistant Professor

Department of Gastroenterology, Jichi Medical University

Associate Professor

Department of Gastroenterology, Jichi Medical University

Fujinon Corporation

Fujinon Corporation

Department of Gastroenterology, Jichi Medical University

Internal Medicine, Nasu Minami Hospital

Department of Gastroenterology, Jichi Medical University

Department of Gastroenterology, Jichi Medical University

Department of Gastroenterology, Jichi Medical University

Department of Gastroenterology, Jichi Medical University

Department of Gastroenterology, Jichi Medical University

Department of Gastroenterology, Jichi Medical University

Department of Gastroenterology, Jichi Medical University 


\section{Contents of Attachment DVD}

The DVD that comes with this book, Procedure Manual for Inserting the

Double-Ballon Endoscope, was provided by Fujinon Corporation. Contents are as follows.

1. Introduction Introduction of the products, preparation, explanation of operation

2. Clinical Examples How to operate the products

3. Interviews Interviews with Dr. Hironori Yamamoto

DVD Planning and creative work : Fujinon Corporation

\begin{tabular}{|c|c|c|c}
\hline \multirow{2}{*}{ VI D E O } & \multicolumn{2}{|c|}{ Stereo sound track recorded on Sony Linear PCM } & \multirow{2}{*}{$\begin{array}{c}\text { Image size } \\
4: 3\end{array}$} \\
\cline { 2 - 3 } & Time : 36 min. & Color & \\
\cline { 2 - 3 } & Single-sided single layer & MPEG2 & \\
\hline
\end{tabular}

